\title{
ON MEASURABLE MULTIFUNCTIONS WITH STOCHASTIC DOMAIN
}

\author{
NIKOLAOS S. PAPAgeORgIOU \\ (Received 8 September 1986; revised 13 May 1987) \\ Communicated by R. O. Vyborny
}

\begin{abstract}
In this paper we prove several random fixed point theorems for multifunctions with a stochastic domain. Then those techniques are used to establish the existence of solutions for random differential inclusions. A useful tool in this process is a stochastic version of the Tietze extension theorems that we prove. Finally we present a stochastic version of the Riesz representation theorem for Hilbert spaces.
\end{abstract}

1980 Mathematics subject classification (Amer. Math. Soc.): primary $60 \mathrm{H} \mathrm{25}$; secondary $34 \mathrm{G}$ 05 .

\section{Introduction}

In this paper we examine single valued and multivalued functions with a stochastic domain. We prove some random fixed point theorems that generalize results existing in the literature. A useful tool in this process is a stochastic version of the Tietze extension theorem. Then, in Section 4, we use the random fixed point theorem to obtain solutions for a random differential inclusion. Finally in Section 5 we examine random linear operators with stochastic domain and we prove a random version of the classical Riesz representation theorem. Our work was motivated from control theory, differential equations and inclusions and mathematical economics, where our results can find useful applications.

This research was supported by N.S.F. Grant D.M.S. 8602313 .

(C) 1988 Australian Mathematical Society $0263-6115 / 88 \$ A 2.00+0.00$ 


\section{Preliminaries}

Let $(\Omega, \Sigma, \mu)$ be a complete $\sigma$-finite measure space and $X$ a Polish space (that is, a complete, separable, metrizable space). We will denote by $d(\cdot, \cdot)$ a metric compatible with the topology of $X$. Recall (see Castaing-Valadier [12] or Himmelberg [4]) that a multifunction $F: 2^{X} \backslash\{\varnothing\}$ is said to be measurable if either of the following two equivalent statements hold:

(1) for all $U \subseteq X$ open, $\{\omega \in \Omega: F(\omega) \cap U \neq \varnothing\} \in \Sigma$,

(2) for all $y \in X, \omega \rightarrow d(y, F(\omega))=\inf [d(y, x): x \in F(\omega)]$ is measurable.

If $F(\cdot)$ is closed valued, then (1) and (2) above are equivalent to (3) $\mathrm{Gr} F=$ $\{\omega, x) \in \Omega \times X: x \in F(\omega)\} \in \Sigma \times B(X), B(X)$ being the Borel $\sigma$-field of $X$ (graph measurability).

Following Engl [11] and Schäl [23] we will say that a measurable $F(\cdot)$ is separable if there exists a countable set $D \subseteq X$ such that $F(\omega)=\overline{F(\omega) \cap D}$ for all $\omega \in \Omega$. It is not difficult to see that if $F($.$) is measurable and F(\omega)=\overline{\operatorname{int} F(\omega)}$, then it is separable. Also a multifunction $T: \operatorname{Gr} F \rightarrow 2^{X} \backslash\{\varnothing\}$ is said to be an "adjective" random map with stochastic domain $F(\cdot)$, if for all $x \in X$ and all $U \subset X$ open, $\{\omega \in \Omega: T(\omega, x) \cap U \neq \varnothing, x \in F(\omega)\} \in \Sigma$ and for every $\omega \in \Omega, x \rightarrow T(\omega, x)$ is "adjective" on $F(\omega)$. Random operators with stochastic domain were first introduced and studied by Engl [8] (single valued case) and [11] (multivalued case).

Recall (see Klein-Thompson [17]), that if $Y, Z$ are Hausdorff topological spaces and $G: Y \rightarrow 2^{Z} \backslash\{\varnothing\}$, then we say that $F(\cdot)$ is upper semicontinuous (u.s.c.) (resp. lower semicontinuous (l.s.c)) if for all $U \subset Z$ open $\{y \in Y: G(y) \subset U\}$ (resp. $\{y \in Y: G(y) \cap U \neq \varnothing\}$ ) is open in $Y$. If $G(\cdot)$ is both u.s.c. and l.s.c., then we say that it is continuous. Also if $Z$ is a metric space and $G(\cdot)$ is closed valued, we say that $G(\cdot)$ is $h$-continuous, if it is continuous from $Y$ into the closed subsets of $Z$ with the Hausdorff (generalized) metric $h(\cdot, \cdot)$. Those two concepts of set valued continuity are equivalent when $G(\cdot)$ is compact valued. Finally for $Z$ a metric space, we will say that $G(\cdot)$ is $d$-continuous if $y \rightarrow d(z, G(y))$ is continuous for all $z \in X$. Here $d(\cdot, \cdot)$ is the metric on $Z$. Note that an $h$-continuous multifunction is $d$-continuous and if $Z$ is a reflexive Banach space, then the same is true for closed, convex valued multifunctions that are continuous in the Kuratowski-Mosco convergence (see Tsukada [26]). This last type of continuity is useful in differential inclusions and variational inequalities.

Let $X$ be a Banach space. We will be using the following notations:

$$
\begin{aligned}
& P_{f(c)}(X)=\{A \subset X: \text { nonempty, closed, (convex) }\} \\
& P_{k(c)}(X)=\{A \subset X: \text { nonempty, compact, (convex) }\}
\end{aligned}
$$


Also by $\gamma(\cdot)$ we will denote the Kuratowski measure of noncompactness, that is, if $A \subset X$ is nonempty and bounded then

$\gamma(A)=\inf \{\varepsilon>0: A$ admits a finite covering of sets of diameter $\leq \varepsilon\}$.

Recall that a map $T: X \rightarrow 2^{X} \backslash\{\varnothing\}$ is said to be condensing if for all $A \subset X$ nonempty, bounded with $\gamma(A)>0$, we have $\gamma(T(A))<\gamma(A)$.

\section{Random fixed point theorems}

In this section we prove the existence of random fixed points for multifunctions with stochastic domain. Throughout this section $(\Omega, \Sigma, \mu)$ is a complete $\sigma$-finite measure space and $X$ a Polish space. Additional hypotheses will be introduced as needed.

We will start with a powerful and general result, that extends to a probabilistic setting a large class of deterministic fixed point theorems. Already such a "general random fixed point theorem" (of the type that a.e. existence of a deterministic fixed point implies existence of a random fixed point) was proved by Engl [9] (Theorem 13) for single valued mappings and $X$ a separable, reflexive Banach space. Then it was extended by Engl [11] (Theorem 13) and [10] (Theorem 6) to multivalued $h$-continuous mappings defined on a separable Banach space. Here we relax the $h$-continuity to $d$-continuity and the space $X$ can be any Polish space. Also our result extends Theorem 4.2 of Cuong [5] and the results of Nowak [19], where a stronger continuity hypothesis was made and Theorem 1 of [21], where the multifunction had a deterministic domain, but the continuity hypotheses were weaker.

THEOREM 3.1. If $F: \Omega \rightarrow P_{f}(X)$ is a separable measurable multifunction, $T: \mathrm{Gr} F \rightarrow P_{f}(X)$ is a d-continuous random map with stochastic domain $F(\cdot)$ and for all $\omega \in \Omega$ there exist $x \in F(\omega)$ such that $x \in T(\omega, x)$ then there exists $x: \Omega \rightarrow X$ measureable, and such that for all $\omega \in \Omega, x(\omega) \in F(\omega)$, and $x(\omega) \in$ $T(\omega, x(\omega))$.

Proof. Let $G(\omega)=F(\omega) \times X$. Since $F(\cdot)$ is separable, measurable, so is $G(\cdot)$. Let $\varphi: \operatorname{Gr} G \rightarrow R_{+}$be defined by $\varphi(\omega, x, y)=d(y, T(\omega, x))$. Note that for $(x, y) \in X \times X$ and for $\lambda>0$ we have: $\{\omega \in \Omega: \varphi(\omega, x, y)<\lambda,(x, y) \in G(\omega\}=$ $\{\omega \in \Omega: T(\omega, x) \cap[z \in X: d(z, y)<\lambda] \neq \varnothing,(x, y) \in G(\omega)\} \in \Sigma$. Also note that for fixed $\omega \in \Omega$, if $\left(x_{n}, y_{n}\right) \rightarrow(x, y)$, then

$$
\begin{aligned}
& \left|d\left(y_{n}, T\left(\omega, x_{n}\right)\right)-d(y, T(\omega, x))\right| \\
& \quad \leq\left|d\left(y_{n}, T\left(\omega, x_{n}\right)\right)-d\left(y, T\left(\omega, x_{n}\right)\right)\right|+\left|d\left(y, T\left(\omega, x_{n}\right)\right)-d(y, T(\omega, x))\right| \\
& \quad \leq d\left(y_{n}, y\right)+\left|d\left(y, T\left(\omega, x_{n}\right)\right)-d(y, T(\omega, x))\right| \rightarrow 0,
\end{aligned}
$$


the summand tending to zero because of the $d$-continuity hypothesis. So $(x, y) \rightarrow$ $\varphi(\omega, x, y)$ is continuous. Thus $\varphi(\cdot, \cdot, \cdot)$ is a continuous random operator with stochastic domain the separable, measurable multifunction $G(\cdot)$. Using Corollary 3.2. of Jdanok [15] we can find $\hat{\varphi}: \Omega \times X \times X \rightarrow R$ a Carathéodory extension of $\varphi(\cdot, \cdot, \cdot)$.

Next let $\hat{T}: \Omega \times X \rightarrow P_{f}(X)$ be the multifunction defined by

$$
\hat{T}(\omega, x)= \begin{cases}T(\omega, x) & \text { for } \omega, x) \in \mathrm{Gr} F, \\ C & \text { for }(\omega, x) \notin \mathrm{Gr} F,\end{cases}
$$

where $C \in P_{f}(X)$ is arbitrary. Consider the multifunction $R: \Omega \rightarrow 2^{X}$ defined by $R(\omega)=\{x \in F(\omega): x \in \hat{T}(\omega, x)\}$. Note that because of our hypothesis, $R(\omega) \neq \varnothing$ for all $\omega \in \Omega$.

Also $\operatorname{Gr} R=\{(\omega, x) \in \operatorname{Gr} F: x \in \hat{T}(\omega, x)\}=\{(\omega, x) \in \operatorname{Gr} F: \hat{\varphi}(\omega, x, x)=$ $0\} \in \Sigma \times B(X)$. So we can apply Aumann's selection theorem (see Saint-Beuve [22], Theorem 3) to find $x: \Omega \rightarrow X$ measurable s.t. $x(\omega) \in R(\omega)$ for all $\omega \in \Omega$. Then $x(\omega) \in F(\omega)$ and $x(\omega) \in T(\omega, x(\omega))$ for all $\omega \in \Omega$.

REMARK. The function $x(\cdot)$ obtained in the above theorem is called a random fixed point for $T(\cdot, \cdot)$.

We will give a small sample of the many useful applications that Theorem 3.1 can have. For the next result assume that $X$ is a reflexive, separable Banach space. By $X_{w}$ we will denote the space $X$ with the weak topology.

THEOREM 3.2. If $F: \Omega \rightarrow P_{f}(X)$ is a separable measurable multifunction, $T: \operatorname{Gr} F \rightarrow P_{f c}(X)$ is a continuous, condensing random multifunction, with stochastic domain $F(\cdot)$ and for all $\omega \in \Omega, T(\omega, F(\omega)) \subset F(\omega)$ and $T(\omega, F(\omega))$ is bounded, then $T(\cdot, \cdot)$ admits a random fixed point.

ProOF. Since $T(\omega, \cdot)$ is continuous from $F(\omega)$ into $X$, it is u.s.c. from $F(\omega)$ into $X_{w}$ and so for $x_{n} \stackrel{s}{\rightarrow} x$ in $F(\omega)$, we have $w$ - $\varlimsup_{n \rightarrow \infty} T\left(\omega, x_{n}\right) \subset T(\omega, x)$ (see Delahaye-Denel [6]). Also $T(\cdot, \cdot)$ is l.s.c. from $F(\omega)$ into $X$ and so $T(\omega, x) \subset s$ $\underline{\lim } T\left(\omega, x_{n}\right)$. Then using Theorem 2.2. of Tsukada [26] we get that $T(\omega, \cdot)$ is $d$-continuous on $F(\omega)$. Finally Theorem 1 of Himmelberg, Porter and Van Vleck [13], together with Theorem 3.1. produce the desired random fixed point.

REMARK. This result extends Theorem 3.1. of Itoh [14], where the multifunction $T(\cdot, \cdot)$ had a deterministic domain and its values were compact, convex sets in $X$.

For the next result assume that $X$ is a separable Banach space. Recall that a multifunction $G: X \rightarrow P_{f}(X)$ is $h$-contractive, if $h\left(G\left(x^{\prime}\right), G(x)\right)<\left\|x^{\prime}-x\right\|$ for all $x^{\prime}, x \in X$. Our result can be viewed as a stochastic version of the fixed point theorem of Assad [1]. 
THEOREM 3.3. If $F: \Omega \rightarrow P_{k}(X)$ is a separable, measurable multifunction, $T: \operatorname{Gr} F \rightarrow P_{f}(X)$ is an $h$-contractive, random operator with stochastic domain $F(\cdot)$ and with bounded values, and for all $(\omega, x) \in \operatorname{Gr}(\operatorname{bd} F(\cdot)), T(\omega, x) \subset F(\omega)$, then $T(\cdot, \cdot)$ admits a random fixed point.

Proof. This follows from Theorem 1.1 of Assad [1] and Theorem 3.1.

REMARK. The above theorem extends Corollary 15 of Engl [11], where $T(\omega, \cdot)$ was a strict contraction, that is, $k(\omega)$-Lipschitz with $k(\omega)<1$ for all $\omega \in \Omega$.

So the strategy is clear. Combine a well known deterministic result with Theorem 3.1. This way we can get several other theorems. In the rest of this section we are going to use a particular version of this method, based on the following interesting extension theorem. The same result was also proved by Bocsan, Constantin and Radu [3]. This theorem is an interesting application of an important extension principle for $R$-valued functions proved by Jdanok [15] under very general hypotheses.

Asume that $X, Y$ are Polish spaces.

THEOREM 3.4. If $F: \Omega \rightarrow P_{f}(X)$ is a separable measurable multifunction, and $T: \mathrm{Gr} F \rightarrow Y$ is a continuous random operator with stochastic domain $F(\cdot)$, then there exists $\hat{T}: \Omega \times X \rightarrow Y$ a Carathéodory map such that $\left.\hat{T}\right|_{\mathrm{Gr} F}=T$.

ProOF. From Urysohn's theorem (see Dugundji [7], page 195, Corollary 9.2) we know that there exists a homeomorphism $u: Y \rightarrow V \subset I^{N}$ where $I=[0,1]$. So we can view $T$ as a map from Gr $F$ into $V$, that is, $T(\omega, x)=$ $\left(T_{1}(\omega, x), T_{2}(\omega, x), \ldots, T_{n}(\omega, x), \ldots\right)$.

We claim that for all $n \geq 1, T_{n}(\cdot, \cdot)$ is a continuous random function with stochastic domain $F(\cdot)$. The continuity is clear from the continuity of $T(\omega, \cdot)$. To show measurability, lelt $p_{n}: I^{N} \rightarrow I$ be the projection on the $n$th factor. We know that $p_{n}(\cdot)$ is a continuous, open surjection. Then for every $x \in F(\omega)$ and for every $U \subset I$ open we have $\left\{\omega \in \Omega: T_{n}(\omega, x) \in U\right\}=\left\{\omega \in \Omega:\left(p_{n} \circ T\right)(\omega, x) \in\right.$ $U, x \in F(\omega)\} \in \Sigma$. For each $n \geq 1$, let $\hat{T}_{n}: \Omega \times X \rightarrow I$ be the Carathéodory extension of $T_{n}$, existing by Corollary 3.2 of Jdanok [15]. Let $\hat{T}: \Omega \times X \rightarrow Y$ be defined by $\hat{T}(\omega, x)=\left(\hat{T}_{1}(\omega, x), \hat{T}_{2}(\omega, x), \ldots, \hat{T}_{n}(\omega, x), \ldots\right)$. Clearly $\hat{T}(\cdot, \cdot)$ is the desired extension of $T(\cdot, \cdot)$.

This result leads us to some other interesting random fixed point theorems for measurable multifunctions with stochastic domain. So let $X$ be a separable Banach space.

THEOREM 3.5. If $F: \Omega \rightarrow P_{f}(X)$ is a separable multifunction, and $T: \mathrm{Gr} F$ $\rightarrow P_{k c}(X)$ is a continuous, bounded, condensing random multifunction with 
stochastic domain $F(\cdot)$ such that for all $\omega \in \Omega, T(\omega, \mathrm{bd} F(\omega)) \subset F(\omega)$, then $T(\cdot, \cdot)$ admits a random fixed point.

ProOF. From Theorem II-14, page 47, of Castaing-Valadier [4], we know that $\left(P_{k c}(Y), h\right)$ is a Polish space. Then Theorem III-2 of [4] tells us that $T(\cdot, \cdot)$ is a continuous, random operator with stochastic domain $F(\cdot)$. Using Theorem 3.4. we can find $\hat{T}: \Omega \times K \rightarrow P_{k c}$, a Carathéodory extension of $T(\cdot, \cdot)$. Let $R(\omega)=$ $\{x \in F(\omega): x \in \hat{T}(\omega, x)\}$. From Corollary 3 of Su-Seghal [25] we know that for all $\omega \in \Omega, R(\omega) \neq \varnothing$. Note that $\operatorname{Gr} R=\{(\omega, x) \in \Omega \times X: d(x, \hat{T}(\omega, x))=0\} \cap \operatorname{Gr} F$. Since measurablity of $\hat{T}(\cdot, x)$ as a function in the metric space $\left(P_{k c}(X), h\right)$ is equivalent to measurablity of $\hat{T}(\cdot, x)$ as a multifunction (see Theorem III-2 in Castaing-Valadier [4]), we see that $(\omega, x) \rightarrow d(x, \hat{T}(\omega, x))$ is a Carathéodory function, that is, is measurable in $\omega$ and continuous in $x$. Hence from Lemma III-14 of [4] we get that $(\omega, x) \rightarrow d(x, \hat{T}(\omega, x))$ is measurable, and thus $\operatorname{Gr} R \in$ $\Sigma \times B(X)$. So we can apply Aumann's selection theorem and get $x: \Omega \rightarrow X$, measurable, such that for all $\omega \in \Omega, x(\omega) \in R(\omega)$. This is the desired random fixed point.

REMARK. The result remains true if instead we assume " $T: \mathrm{Gr} F \rightarrow P_{f c}(X)$ is a continuous, bounded, condensing random multifunction with stochastic domain $F(\cdot)$ and with values in a separable subset $C$ of the space of nonempty, bounded, closed, convex subsets of $X$ with the Hausdorff metric".

The proof is the same, if we consider the Polish space $(C, h)$. Similarly, using Theorem 1 of Su-Seghal [25] we can have

THEOREM 3.6. If $F(\cdot)$ is as above, and $T: \operatorname{Gr} F \rightarrow P_{k c}(X)$ is a continuous, bounded, condensing random multifunction such that for all $(\omega, x) \in$ $\operatorname{Gr} F, T(\omega, x) \cap F \neq \varnothing$, then $T(\cdot, \cdot)$ admits a random fixed point

REMARKs. (1) Again we can assume instead that $T: \operatorname{Gr} F \rightarrow P_{f c}(X)$ is a continuous, bounded, condensing random multifunction with values in $C$.

(2) This result extends Theorem 2 of Sehgal and Walters [24], which was stated for single valued functions with domain $\Omega \times X$.

Also Theorem 3.4. leads us to the following implicit function theorem of Filippov type, that can be useful in control theory and mathematical economics. Assume that $X, Y$ are Polish spaces.

THEOREM 3.7. If $F: \Omega \rightarrow P_{f}(X)$ is a separable, measurable multifunction, $T: \mathrm{Gr} F \rightarrow P_{k}(Y)$ is a continuous, random multifunction with stochastic domain $F(\cdot), G: \Omega \rightarrow 2^{X} \backslash\{\varnothing\}$ is graph measurable and for all $\omega \in G, G(\omega) \subset F(\omega)$ and if $f: \Omega \rightarrow Y$ is measurable such that for all $\omega \in \Omega, f(\omega) \in T(\omega, G(\omega))$, then 
there exists $g: \Omega \rightarrow X$ measurable such that for all $\omega \in \Omega, g(\omega) \in G(\omega)$ and $f(\omega) \in T(\omega, g(\omega))$.

PrOOF. From Theorem II-8, p. 42 of Castaing and Valadier [4] we know that $\left(P_{k}(Y), h\right)$ is a Polish space. Apply Theorem 3.4. to find $\hat{T}: \Omega \times X \rightarrow$ $P_{k}(Y)$, a Carathéodory extension of $T(\cdot, \cdot)$. Let $R: \Omega \rightarrow 2^{X}$ be defined by $R(\omega)=\{x \in G(\omega): f(\omega) \in \hat{T}(\omega, x)\}$. Clearly for all $\omega \in \Omega, R(\omega) \neq \varnothing$. Also $\operatorname{Gr} R=\{(\omega, x) \in \operatorname{Gr} G: d(f(\omega), \hat{T}(\omega, x))=0\}$. But recall that $(\omega, x) \rightarrow$ $d(f(\omega), \hat{T}(\omega, x))$ is a Carathéodory function, and hence it is jointly measurable. Therefore $\operatorname{Gr} R \in \Sigma \times B(X)$. Once Again Aumann's selection theorem gives us $g: \Omega \rightarrow X$ measurable such that for all $\omega \in \Omega, g(\omega) \in R(\omega)$ implies $g(\omega) \in G(\omega)$ and $f(\omega) \in T(\omega, g(\omega))$.

REMARK. Again we can assume instead that " $T: \operatorname{Gr} F \rightarrow P_{f}(X)$ is a continuous, random multifunction with stochastic domain $F(\cdot)$ and values in a separable subset $C^{\prime}$ of the set of all bounded, closed subsets of $X^{\prime \prime}$

Interesting random fixed point theorems for u.s.c. operators can be found in [10] and [11].

\section{A random differential inclusion}

In this section, using a random fixed point argument, we will establish the existence of solutions for a class of random differential inclusions defined in a separable Banach space.

Our existence theorem extends to infinite dimensions the work of Nowak [20]. Also the orientor field in our case satisfies weaker continuity hypotheses (Carathéodory conditions) and a more general growth assumption. When specialized to single valued functions, our result extends significantly Theorem 4.3 of Itoh [14].

So let $(\Omega, \Sigma, \mu)$ be a complete, $\sigma$-finite measure space, $T=[0, b]$ a bounded, closed interval in $R_{+}$, and $X$ a separable Banach space. Given $F: \Omega \times T \times$ $X \rightarrow 2^{X} \backslash\{\varnothing\}$ and $x_{0}: \Omega \rightarrow X$ measurable, we consider the following random multivalued Cauchy problem:

$$
\dot{x}(\omega, t) \in F(\omega, t, x(\omega, t)), \quad x(\omega, 0)=x_{0}(\omega) .
$$

By a random solution of $(*)$ we understand process $x: \Omega \times T \rightarrow X$ which is measurable in $\omega$, absolutely continuous in $t$ and satisfies (*) for all $\omega \in \Omega$ and almost all $t \in T$. We will make the following assumptions concerning $F(\cdot, \cdot, \cdot)$ :

$\left(\mathrm{A}_{1}\right) F(\cdot, \cdot, \cdot)$ has values in $P_{k c}(Y)$.

$\left(\mathrm{A}_{2}\right)$ for all $x \in X,(\omega, t) \rightarrow F(\omega, t, x)$ is measurable.

$\left(\mathrm{A}_{3}\right)$ for all $(\omega, t) \in \Omega \times T, x \rightarrow F(\omega, t, x)$ is $h$-continuous. 
$\left(\mathrm{A}_{4}\right)|F(\omega, t, x)|=\sup \{\|z\|: z \in F(\omega, t, x)\} \leq a(\omega, t)+b(\omega, t)\|x\|$ a.e. for all $\omega \in \Omega$ and all $x \in X$, where $a(\cdot, \cdot)$ and $b(\cdot, \cdot)$ are jointly measurable, and for all $\omega \in \Omega, a(\omega, \cdot), b(\omega, \cdot) \in L_{+}^{1}$.

$\left(\mathrm{A}_{5}\right)$ For all $B \subset X$ bounded, $\gamma[F(\omega, t, B)] \leq \varphi(\omega, t) \gamma(B)$ a.e. for all $\omega \in$ $\Omega$, where $\varphi(\cdot, \cdot)$ is jointly measurable and for all $\omega \in \Omega, \varphi(\omega, \cdot) \in L_{+}^{1}$ and $\|\varphi(\omega, \cdot)\|_{1}<1 / 2$.

THEOREM 4.1. If $\left(\mathrm{A}_{1}\right)$ to $\left(\mathrm{A}_{5}\right)$ hold then $(*)$ admits a random solution.

Proof. Let $M(\omega)=\left[\left\|x_{0}(\omega)\right\|+\|a(\omega, \cdot)\|_{1}\right] \exp \left[\|b(\omega, \cdot)\|_{1}\right]$. Let $B(\omega) \subseteq$ $C(T, X)$ be defined by $B(\omega)=\left\{x(\cdot) \in C(T, X):\|x(\cdot)\|_{\infty} \leq M(\omega)\right\}$.

Let $R: \Omega \times C(T, X) \rightarrow 2^{C(T, X)}$ be defined by $R(\omega, x)=\{y(\cdot) \in C(T, X): y(t)$ $=x_{0}(\omega)+\int_{0}^{t} f(s) d s, t \in T, f(\cdot) \in L^{1}(T, X), f(s) \in F(\omega, s, x(s))$ a.e. $\}$.

Note that because of Aumann's selection theorem and $\left(\mathrm{A}_{4}\right)$ it is easy to see that $R(\omega, x) \neq \varnothing$. Next we will show that $\omega \rightarrow R(\omega, x)$ is measurable, while $x \rightarrow R(\omega, x)$ is $h$-continuous.

Let $\int_{0}^{t} F(\omega, s, x(s)) d s=\left\{\int_{0}^{t} f(s) d s: f(\cdot) \in L^{1}(T, X), f(s) \in F(\omega, s, x(s))\right.$ a.e. $\}$. As we already said this is nonempty and because of the Rädstrom embedding theorem (see Klein and Thompson [17] it is easy to see that

$$
\int_{0}^{t} F(\omega, s, x(s)) d s \in P_{k c}(X) \text { for all } t \in T .
$$

So a straightforward application of the Arzela and Ascoli theorem tells us that $R(\cdot, \cdot)$ has values in $P_{k c}(C(T, X))$. Now note that $\operatorname{Gr} R=\{(\omega, x, y) \in \Omega \times$ $C(T, X) \times C(T, X) ; d(y(t), L(\omega, t, x))=0$ for all $t \in T\}$, where $L(\omega, t, x)=$ $x_{0}(\omega)+\int_{0}^{t} F(\omega, s, x(s)) d s$. Let $u(\omega, t, x, y)=x_{0}(\omega)+d\left(e_{t}(y), L(\omega, t, x)\right)$ where $e_{t}: C(T, X) \rightarrow X$ is the evaluation map at $t$. From Theorem 2.4, page 260 of Dugundji [7] we know that $e .(\cdot)$ is continuous. Also from $\left(\mathrm{A}_{2}\right) \omega \rightarrow L(\omega, t, x)$ is measurable. Furthermore if $\left(t_{n}, x_{n}\right) \rightarrow(t, x)$ in $T \times C(T, X)$, then using $\left(\mathrm{A}_{3}\right)$ we have

$$
\begin{aligned}
h\left(\int_{0}^{t_{n}}\right. & \left.F\left(\omega, s, x_{n}(s)\right) d s, \int_{0}^{t} F(\omega, s, x(s)) d s\right) \\
\leq & h\left(\int_{0}^{t_{n}} F\left(\omega, s, x_{n}(s)\right) d s, \int_{0}^{t_{n}} F(\omega, s, x(s)) d s\right) \\
& +h\left(\int_{0}^{t_{n}} F(\omega, s, x(s)) d s, \int_{0}^{t} F(\omega, s, x(s)) d s\right) \\
\leq & \int_{0}^{t_{n}} h\left(F\left(\omega, s, x_{n}(s)\right), F(\omega, s, x(s))\right) d s \\
& +\int_{t \wedge t_{n}}^{t \vee t_{n}}|F(\omega, s, x(s))| d s \rightarrow 0, \quad \text { as } n \rightarrow \infty .
\end{aligned}
$$


Hence $(t, x) \rightarrow L(\omega, t, x)$ is $h$-continuous, so $(\omega, t, x, y) \rightarrow u(\omega, t, x, y)$ is measurable in $\omega$ and continuous in $(t, x, y)$, whence $u(\cdot, \cdot, \cdot, \cdot)$ is jointly measurable, and so $(\omega, x, y) \rightarrow v(\omega, x, y)=\sup _{n \geq 1} u\left(\omega, t_{n}, x, y\right)$ is measurable, where $\left\{t_{n}\right\}_{n \geq 1}$ is dense in $T$. But $\operatorname{Gr} R=\{(\omega, x, y) \in \Omega \times C(T, X) \times C(T, X): v(\omega, x, y)=0\} \in$ $\Sigma \times B(C(T, X)) \times B(C(T, X))$ implies that $R(\cdot, \cdot)$ is graph measurable.

Next let $S_{F(\omega, r, z(\cdot))}^{1}=\left\{f(\cdot) \in L^{1}(T, X): f(s) \in F(\omega, s, z(s))\right.$ a.e. $\}$. Also let $x_{n}(\cdot) \rightarrow x(\cdot)$ in $C(T, X)$. Then using Hörmander's formula (see Theorem II-18, page 49, of Castaing and Valadier [4]), we have that (recall $\sigma\left(x^{*}, A\right)=$ $\sup \left[\left(x^{*}, a\right), a \in A\right]$ for $A \subset X$ and $\left.x^{*} \in X^{*}\right)$ :

$$
\begin{aligned}
& h\left(S_{F\left(\omega, \cdot, x_{n}(\cdot)\right)}^{1}, S_{F(\omega, \cdot, x(\cdot))}^{1}\right) \\
& \quad=\sup _{\|v\|_{\infty} \leq \infty}\left|\sigma\left(v, S_{F\left(\omega, \cdot, x_{n}(\cdot)\right)}^{1}\right)-\sigma\left(v, S_{F(\omega, \cdot, x(\cdot))}^{1}\right)\right| \\
& \quad \leq \sup _{\|v\|_{\infty} \leq 1} \int_{0}^{t}\left|\sigma\left(v(s), F\left(\omega, s, x_{n}(s)\right)\right)-\sigma(v(s), F(\omega, s, x(s)))\right| d s \\
& \quad \leq \int_{0}^{t} h\left(F\left(\omega, s, x_{n}(s)\right), F(\omega, s, x(s))\right) d s \rightarrow 0, \quad \text { as } n \rightarrow \infty,
\end{aligned}
$$

and so

$$
S_{F\left(\omega, \cdot, x_{n}(\cdot)\right)}^{1} \stackrel{h}{\rightarrow} S_{F(\omega, \cdot, x(\cdot))}^{1} \quad \text { as } n \rightarrow \infty .
$$

Let $z(\cdot) \in R(\omega, x)$. Then

$$
z(t)=x_{0}(\omega)+\int_{0}^{t} f(s) d s, \quad t \in T
$$

with $f(\cdot) \in S_{F(\omega,, x(\cdot))}^{1}$. Let $f_{n}(\cdot) \in S_{F\left(\omega,, x_{n}(\cdot)\right)}^{1}$ be such that $f_{n} \stackrel{s}{\rightarrow} f$ in $L^{1}(X)$. This is possible because of (1). Set

$$
z_{n}(t)=x_{0}(\omega)+\int_{0}^{t} f_{n}(s) d s, \quad t \in T .
$$

Clearly $z_{n}(\cdot) \in R\left(\omega, x_{n}\right)$ and

$$
\left\|z_{n}-z\right\|_{\infty} \leq \int_{0}^{b}\left\|f_{n}(s)-f(s)\right\| d s \rightarrow 0, \quad \text { as } n \rightarrow \infty,
$$

so $z \in s-\underline{\lim }_{n \rightarrow \infty} R\left(\omega, x_{n}\right)$, whence

$$
R(\omega, \cdot) \text { is l.s.c. }
$$

(see Delahaye and Denel [6]).

Next we will show that $R(\omega, \cdot)$ is u.s.c. Because it has compact values it suffices to show that given $\varepsilon>0$ there exists $\delta(\varepsilon, x)>0$ such that $R(\omega, y) \subseteq$ $R(\omega, x)+\varepsilon B_{1}$ for all $y \in B_{\delta}(x)$, where $B_{\delta}(x)=\left\{z(\cdot) \in C(T, X):\|z-x\|_{\infty}<\delta\right\}$ and $B_{1}$ is the open unit ball. Recall that $x(\cdot) \rightarrow S_{F(\omega, \cdot, x(\cdot))}^{1}$ is $h$-continuous. So 
we can find $\delta>0$ such that for $\|x-y\|_{\infty}<\delta$ we have $h\left(S_{F(\omega,, x(\cdot))}^{1} S_{F(\cdot, y(\cdot), \cdot)}^{1}\right)<\varepsilon$. Our claim is that this $\delta>0$ will do the job for us. So let $z \in R(\omega, y)$. Then

$$
\begin{aligned}
d(z, R(\omega, x)) & =\inf _{f \in S_{F(\omega, \cdot, x(\cdot))}^{1}} \sup _{t \in T}\left\|z(t)-x_{0}(\omega)-\int_{0}^{t} f(s) d s\right\| \\
& =\inf _{f \in S_{F(\omega, \cdot, x(\cdot))}^{1}} \sup _{t \in T}\left\|x_{0}(\omega)+\int_{0}^{t} g(s) d s-x_{0}(\omega)-\int_{0}^{t} f(s) d s\right\|
\end{aligned}
$$

where $g(\cdot) \in S_{F(\omega, \cdot, y(\cdot))}^{1}$ and $z(t)=x_{0}(\omega)+\int_{0}^{t} g(s) d s, t \in T$. So we have

$$
\begin{aligned}
d(z, R(\omega, x)) & =\inf _{f \in S_{F(\omega, \cdot, x(\cdot))}^{1}}\left\|\int_{0}^{t} g(s) d s-\int_{0}^{t} f(s) d s\right\| \\
& \leq \inf _{f \in S_{F(\omega, \cdot, x(\cdot))}^{1}} \int_{0}^{t}\|g(s)-f(s)\| d s=d\left(g, S_{F_{(\omega,, x(\cdot))}^{1}}\right)<\varepsilon,
\end{aligned}
$$

whence $R(\omega, y) \subseteq R(\omega, x)+\varepsilon B_{1}$, and so

$$
R(\omega, \cdot) \text { is u.s.c. }
$$

From (2) and (3) we conclude tht $R(\omega, \cdot)$ is continuous and because it has compact values it is $h$-continuous.

Next we will show that for each $\omega \in \Omega R(\omega, \cdot)$ has a fixed point in $B(\omega)$. To this end note that because of Gronwall's inequality, for all $x(\cdot) \in B(\omega), R(\omega, x) \subseteq$ $B(\omega)$. So $R(\omega, \cdot): B(\omega) \rightarrow B(\omega)$. Next let $A \subseteq B(\omega)$ be nonempty. We have

$$
\gamma[R(\omega, A)(t)] \leq \gamma\left[\int_{0}^{t} F(\omega, s, A(s)) d s\right] .
$$

Let $\left\{x_{n}(\cdot)\right\}_{n \geq 1} \subseteq B(\omega)$ be such that $\operatorname{cl}\left\{x_{n}(\cdot)\right\}_{n \geq 1}=\bar{A}$. This set exists because $\bar{A}$ being a closed subset of the separable Banach space $C(T, X)$ is itself separable. We claim that, for all $s \in T$,

$$
\operatorname{cl}[F(\omega, s, \bar{A}(s))]=\operatorname{cl}\left\{F\left(\omega, s, x_{n}(s)\right)\right\}_{n \geq 1} .
$$

So let $y \in F(\omega, s, \bar{A}(s))$; then $y \in F(\omega, s, z)$ with $z \in \bar{A}(s)$. Let $\left\{x_{m}(\cdot)\right\}_{m \geq 1} \subseteq$ $\left\{x_{n}(\cdot)\right\}_{n \geq 1}$ be such that $x_{m}(s) \stackrel{\oplus}{\rightarrow} z$; then $F\left(\omega, s, x_{m}(s)\right) \stackrel{h}{\rightarrow} F(\omega, s, z)$. Let $y_{m} \in F\left(\omega, s, x_{m}(s)\right)$ be such that

$$
\left\|y_{m}-y\right\|=d\left(y, F\left(\omega, s, x_{m}(s)\right)\right) \text {. }
$$

Then $\left\|y_{m}-y\right\| \rightarrow 0$ as $m \rightarrow \infty$, so $y \in \operatorname{cl}\left\{F\left(\omega, s, x_{n}(s)\right)\right\}_{n \geq 1}$, and so the claim follows. But note that because of $\left(A_{2}\right)$ and $\left(A_{3}\right) s \rightarrow F\left(\omega, s, x_{n}(s)\right)$ is measurable implies $s \rightarrow \overline{\text { conv }} \bigcup_{n \geq 1} F\left(\omega, s, x_{n}(s)\right)=H(\omega, s)$ is measurable. So it admits a Castaing representation $\left\{h_{n}(\omega, \cdot)\right\}_{n \geq 1}$ (see Castaing-Valadier [4], Theorem III-7, 
page 66). Using it we have that

$$
\begin{gathered}
\operatorname{cl} \int_{0}^{t} \overline{\operatorname{conv}}\left\{h_{n}(\omega, s)\right\}_{n \geq 1} d s=\int_{0}^{t} \overline{\operatorname{conv}}\left\{h_{n}(\omega, s)\right\}_{n \geq 1} d s \\
=\operatorname{cl} \int_{0}^{t}\left\{h_{n}(\omega, s)\right\}_{n \geq 1} d s
\end{gathered}
$$

which implies that

$$
\gamma\left[\int_{0}^{t} F(\omega, s, A(s)) d s\right] \leq \gamma\left[\int_{0}^{t} h_{n}(\omega, s) d s: n \geq 1\right] .
$$

Using Lemma 2.2. of Kisielewicz [16] we get that

$$
\begin{aligned}
\gamma\left[\int_{0}^{t} h_{n}(\omega, s) d s: n \geq 1\right] & \leq \int_{0}^{t} 2 \gamma\left[h_{n}(\omega, s): n \geq 1\right] d s \\
& =\int_{0}^{t} 2 \gamma(F(\omega, s, \bar{A}(s))) d s \\
& \leq \int_{0}^{t} 2 \varphi(\omega, s) \gamma(\bar{A}(s)) d s
\end{aligned}
$$

Let $\psi(\cdot)$ denote the Kuratowski measure of noncompactness on $C(T, X)$. From a well-known result of Ambrosetti (see for example Lakshmikantham and Leela [18]) we have $\psi(R(\omega, A)) \leq 2 \psi(A)\|\varphi(\omega, \cdot)\|_{1}<\psi(A)$, whence $R(\omega, \cdot)$ is $\psi$-condensing.

Apply Theorem 1 of Himmelberg-Porter-Van Vleck [13] to deduce that there exists $x: \Omega \rightarrow C(T, X)$ measurable, and such that $x(\omega) \in B(\omega)$ and $x(\omega) \in$ $R(\omega, x(\omega))$ for all $\omega \in \Omega$. Because of Proposition 4.2. of Itoh [14] (see also the Lemma in [21]) we have that $x(\omega, t)=x(\omega)(t)$ is a Carathéodory function and clearly is the desired random solution of $(*)$.

\section{Random linear functionals}

In this final section of the paper we prove a random version of the Riesz representation theorem for Hilbert spaces.

So let $(\Omega, \Sigma, \mu)$ be a complete, $\sigma$-finite measure space and $X$ a separable Hilbert space.

THEOREM 5.1. If $L: \Omega \rightarrow P_{f}(X)$ is a separable, measurable multifunction with values in the closed subspaces of $X$ and $T: \operatorname{Gr} L \rightarrow R$ is a linear, continuous, random functional with stochastic domain $L(\cdot)$, then there exists a unique $x^{*}: \Omega \rightarrow X$, measurable, and such that for all $\omega \in \Omega, x^{*}(\omega) \in L(\omega)$, $T(\omega, x)=\left(x^{*}(\omega), x\right)$ for all $x \in L(\omega)$, and $\|T(\omega, \cdot)\|=\left\|x^{*}(\omega)\right\|$. 
PROOF. From Corollary 4.4. of Jdanok [15] we know that there exists $\hat{T}: \Omega \times$ $X \rightarrow R$, a linear, continuous, measurable functional such that $\left.\hat{T}\right|_{\mathrm{Gr} L}=T$ and $\|\hat{T}(\omega)\|=\|T(\omega)\|$ for all $\omega \in \Omega$.

Let $R: \Omega \rightarrow 2^{X}$ be defined by

$$
R(\omega)=\left\{x^{*} \in L(\omega):\left(x^{*}, x\right)=\hat{T}(\omega, x) \text { for all } x \in L(\omega)\right\} .
$$

Since $L(\omega)$ is a Hilbert space itself, from the classical Riesz representation theorem we know that $R(\omega) \neq \varnothing$ for all $\omega \in \Omega$. Let $\left\{x_{n}(\cdot)\right\}_{n \geq 1}$ be a Castaing representation for $L(\cdot)$; that is, for all $n \geq 1, x_{n}: \Omega \rightarrow X$ is measurable and $L(\omega)=\operatorname{cl}\left\{x_{n}(\omega)\right\}_{n \geq 1}$. Then $\operatorname{Gr} R=\bigcap_{n \geq 1}\left\{\left(\omega, x^{*}\right) \in \Omega \times X:\left(x^{*}, x_{n}(\omega)\right)-\right.$ $\left.\hat{T}\left(\omega, x_{n}(\omega)\right)=0\right\} \cap \operatorname{Gr} L$. Note that for all $n \geq 1, u_{n}\left(\omega, x^{*}\right)=\left(x^{*}, x_{n}(\omega)\right)-$ $\hat{T}\left(\omega, x_{n}(\omega)\right)$ is jointly measurable and so $\operatorname{Gr} R \in \Sigma \times B(X)$. Apply Aumann's selection theorem to find $x^{*}: \Omega \rightarrow X$ measurable such that $x^{*}(\omega) \in R(\omega)$ for all $\omega \in \Omega \Rightarrow x^{*} \in L(\omega)$ and $\left(x^{*}(\omega), x\right)=T(\omega, x)$ for all $x \in L(\omega)$ and all $\omega \in \Omega$. From this last equality we also get that $\left\|x^{*}(\omega)\right\|=\|T(\omega)\|$ for all $\omega \in \Omega$.

REMARK. If for all $\omega \in \Omega, L(\omega)=X$, then we recover the result of Bensoussan [2], page 89, which is useful in filtering theory.

\section{Acknowledgment}

The author would like to express his deep gratitude to the two referees for their invaluable comments and suggestions, that helped improve the presentation of the material considerably.

\section{References}

[1] N. Assad, 'Fixed point theorems for set valued transformations on compact sets', Boll. Un. Mat. Ital. 8 (1973), 1-7.

[2] A. Bensoussan, Filtrage optimal des systèmes linéaires (Dunod, Paris, 1971).

[3] Gh. Bocsan, Gh. Constantin and V. Radu, 'On the random extension property at a separable metric space', Sem. de Teoria Prob. si Appl. 53 (1980), Univ. Timisoara (Romania) pp. 1-5.

[4] C. Castaing and M. Valadier, Convex analysis and measurable multifunctions (Lecture Notes in Math., vol. 580, Berlin, 1977).

[5] P. V. Cuong, 'Random versions of Kakutani-Ky Fan's fixed point theorems', J. Math. Anal. Appl. 82 (1981), 473-490.

[6] J. P. Delahaye and J. Denel, 'The continuities of the point-to-set maps, definitions and equivalences', Math. Programming Study 10 (1979), 8-12.

[7] J. Dugundji, Topology (Allyn and Bacon, Boston, Mass., 1966). 
[8] H. W. Engl, 'Some random fixed point theorems for strict contractions and nonexpansive mappings', Nonlinear Anal. 2 (1978), 619626.

[9] H. W. Engl, 'A general stochastic fixed point theorem for continuous random operators with stochastic domains', J. Math. Anal. Appl. 66 (1978), 220-231.

[10] H. W. Engl, 'Random fixed point theorems', Nonlinear equations in abstract spaces, V. Lakshimikantham (ed.), (Academic Press, 1980, pp. 67-80.)

[11] H. W. Engl, 'Random fixed point theorems for multivalued mappings', Pacific J. Math 76 (1978), 351-360.

[12] C. Himmelberg, 'Measurable relations', Fund. Math. 87 (1975), 53-72.

[13] C. Himmelberg, J. Porter and F. Van Vleck, 'Fixed point theorems for condensing multifunctions', Proc. Amer. Math. Soc. 23 (1969), 635-641.

[14] S. Itoh, 'Random fixed point theorems with an application to random differential equations in Banach spaces', J. Math. Anal. Appl. 67 (1979), 261-272.

[15] T. Jdanok, 'Operateurs et fonctionelles aleatoires dans les champs mesurables', Seminaire d'analyse convexe, expose 2, Montpellier (1983), 1-35.

[16] M. Kisielewicz, 'Multivalued differential equations in separable Banach spaces', J. Optim. Theory Appl. 37 (1982), 231-249.

[17] E. Klein and A. Thompson, Theory of correspondences (Wiley, New York, 1984).

[18] V. Lakshmikantham and S. Leela, Nonlinear differential equations in abstract spaces (Pergamon Press, London, 1981).

[19] A. Nowak, 'Random fixed point of multifunctions', Prace Nauk. Uniw. Slask. Katowic. 11 (1981), 36-41.

[20] A. Nowak, 'Applications of random fixed point theorems in the theory of generalized random differential equations', Bull. Acad. Pol. Sci. Ser. Math. 34 (1986), 487-494.

[21] N. S. Papageorgiou, 'Random fixed point theorems for measurable multifunction in Banach spaces', Proc. Amer. Math. Soc. 97 (1986), 507-514.

[22] M. F. Saint-Beuve, 'On the extension of Von Neumann-Aumann's theorem', J. Funct. Anal. 17 (1974), 112-129.

[23] M. Schäl, 'A selection theorem for optimization problems', Arch. Math. 25 (1974), 219224.

[24] V. M. Seghal and C. Waters, 'Some random fixed point theorems for considering operators', Proc. Amer. Math. Soc. 90 (1984), 425-429.

[25] Ch. H. Su and V. M. Seghal, 'Some fixed point theorems for condensing multifunctions in locally convex spaces', Proc. Amer. Math. Soc. 50 (1975), 150-154.

[26] M. Tsukada, 'Convergence of best approximations in smooth Banach spaces', J. Approximation Theory 40 (1984), 301-309.

\author{
Department of Mathematics \\ University of California \\ Davis, California 95616 \\ U.S.A.
}

